\title{
Aplicando Mensuração em Microempresas de Software para Suporte da Gerência de Projetos
}

\author{
Alessandra Anacleto ${ }^{1}$, Christiane Gresse von Wangenheim ${ }^{2}$ \\ ${ }^{1}$ Universidade Federal de Santa Catarina, Trindade, 88049-200 Florianópolis, Brasil \\ ale@inf.ufsc.br \\ ${ }^{2}$ Universidade do Vale do Itajaí/CES VII \\ Rod. SC 407, Km 04,CEP 88122-000 São José/SC, Brasil \\ gresse@sj.univali.br
}

\begin{abstract}
Resumo
Microempresas de software em geral sofrem com problemas de limitações de recursos e pessoal, além de não utilizarem sistematicamente técnicas de desenvolvimento e padrões para o gerenciamento de seus negócios, o que freqüentemente resulta em problemas de qualidade e produtividade. Uma das infra-estruturas essenciais para analisar os pontos fracos e identificar os problemas na gerência dos projetos de software é a mensuração. O que dificulta a aplicação de métricas em microempresas é a falta de uma abordagem adaptada às suas necessidades. Para facilitar a aplicação de mensuração neste setor no que se refere à gerência de projetos foi desenvolvido um modelo de mensuração baseado na abordagem GQM (Goal/Question/Metric). Este modelo tem por base a análise e comparação de experiências ganhas em estudos de caso planejados e executados em três microempresas de software. Ele auxilia no planejamento de um programa de mensuração enfocando na gerência de projetos e fornece guias para adaptação a um contexto específico.
\end{abstract}

Palavras-Chave: Mensuração, gerência de projetos, microempresa de software

\section{Abstract}

Small software companies, in general, are restricted by limited resources besides the fact that they do not use systematically techniques and standards for the management of their businesses, which frequently results in problems related to quality and productivity. An essential infrastructure in order to analyze weaknesses and to identify improvement oportunities is measurement. What makes the application of metrics difficult in small companies is the lack of an adapted approach considering their specific needs. In order to facilitate its application in this specific sector with respect to the management of software projects, a measurement model has been developed based on the GQM (Goal/Question/Metric) approach. This model is based on the analysis and comparison of experiences gained through case studies in three small software companies. The model supports the planning of a measurement program focusing on project management and provides guidelines for its adaptation in a specific context.

Key-words: Measurement, project management, small software company

O presente trabalho foi realizado com o apoio do CNPq, uma entidade do Governo Brasileiro voltada ao desenvolvimento científico e tecnológico.

\section{Introdução}

O final da década de sessenta acompanhou a então chamada "crise do software", fortemente marcada pela desestruturação de seus desenvolvedores, que não seguiam padrões nem regras para implementar. Esse panorama era ainda agravado pelo setor de hardware, que encontrava-se em pleno desenvolvimento, e necessitava de software mais eficiente para operá-lo. Mas o setor de software não conseguia (e até hoje não conseguiu) alcançar a velocidade de evolução do hardware. Foi neste cenário que a engenharia de software teve seu início marcado. Era preciso estabelecer padrões, técnicas organizacionais, e até mesmo de 
implementação para tentar suprir os problemas que o software enfrentava. Ainda hoje, projetos de software são atrasados e têm problemas de custo, por falta de um planejamento adequado e de controle sistemático. Esse problema acarreta muitas vezes o fracasso do projeto.

A obtenção de produtos de sucesso depende não somente de uma equipe técnica competente, mas também de pessoal especializado em questões gerenciais. Tradicionalmente, os profissionais de software dão extrema importância a questões técnicas deixando de lado aspectos gerenciais, como o controle sistemático dos projetos em andamento. Para obter software mais confiáveis, com menos erros, feitos dentro dos prazos estabelecidos e com os custos planejados, precisa-se de alguma forma mapeá-los para determinar os pontos fortes e fracos no seu processo de desenvolvimento e assim trabalhar diretamente sobre os problemas que ocorrem valorizando o que já é eficiente. Somente com a sistematização do processo de desenvolvimento é possível melhorar a qualidade do software continuamente e deixar o resultado (o produto) mais previsível. Esta situação é ainda mais evidente no caso de microempresas de software.

\section{Microempresas de Software}

As microempresas de software fazem parte de um mercado crescente no setor de informática atual. Apesar disso, elas ainda mostram um perfil muito imaturo nos processos de desenvolvimento utilizados e na qualidade de seu software. Além de sofrerem com falta de recursos e pessoal, estas empresas na maioria não utilizam técnicas de desenvolvimento, nem têm padrões de processos bem definidos. O resultado são produtos entregues fora do prazo, software com grande margem de erros, baixa usabilidade e consequentemente clientes descontentes. De acordo com a Pesquisa Nacional de Qualidade e Produtividade no Setor de Software Brasileiro [6], de 30\% a 35\% das microempresas pesquisadas possuem atividades de gerência.

Nesse contexto a mensuração é uma infra-estrutura essencial como base para o gerenciamento sistemático de projetos e para atingir o melhoramento sistemático da produtividade e da qualidade do software. Nos últimos anos têm sido desenvolvidas algumas técnicas que auxiliam no processo de aplicação dos programas de mensuração. Contudo, as pequenas e, principalmente, as microempresas não contam com técnicas que as auxiliem na aplicação de mensuração. Os modelos desenvolvidos são voltados para empresas de maior porte e dificilmente são adaptáveis para microempresas. Destas técnicas, a abordagem GQM (Goal/Question/Metric), é a que mais tem sido utilizada com sucesso na prática. Esta abordagem é utilizada para a definição e análise de metas operacionais e mensuráveis. Porém, ela deve ser feita sempre desde as fases iniciais e também não fornece nenhum suporte específico para sua utilização em microempresas considerando as limitações e características deste tipo de empresas. De acordo com [6], das microempresas que utilizam algum método para prevenção de defeitos, apenas 4,8\% utilizam métricas. Como este é um grande setor da indústria de informática, e ainda está em crescimento, é necessária a criação de modelos que permitam a essas empresas melhorar seus processos de desenvolvimento garantindo assim a qualidade de seus produtos de software e o seu sucesso no mercado. No intuito de suprir em parte esta falta de tecnologias adaptadas ao contexto de microempresas, neste trabalho, é desenvolvido um modelo de mensuração que tem por objetivo viabilizar a aplicação de 
mensuração para o suporte da gerência de projetos em microempresas de software com base na abordagem GQM orientada a metas adaptada às necessidades deste setor.

\section{Estudo de Caso Replicado Aplicando Mensuração em Microempresas de Software}

Para criar uma base para desenvolver o modelo é feito um estudo de caso replicado incluindo planejamento e execução de programas de mensuração em três microempresas de software. $\mathrm{O}$ estudo de caso replicado é planejado seguindo a metodologia de estudos de caso da Engenharia de Software [4] baseado na abordagem de melhoria da qualidade, o Quality Improvement Paradigm [8] (veja Figura 1).

\begin{tabular}{|c|c|c|}
\hline \multicolumn{2}{|c|}{1 Caracterizar o contexto } & \\
\hline \multicolumn{2}{|c|}{2 Definir metas do caso estudado } & \\
\hline \multicolumn{3}{|l|}{3 Selecionar modelos } \\
\hline \multirow{3}{*}{$\begin{array}{l}4 \text { Executar os estudos } \\
\text { de caso }\end{array}$} & Programa GQM Making & \multirow{3}{*}{$\begin{array}{l}\text { Incubadora } \\
\text { Centro GeNESS }\end{array}$} \\
\hline & Programa GQM Canal Publicitário & \\
\hline & Programa GQM IASoft & \\
\hline \multicolumn{2}{|c|}{5 Análise dos casos estudados } & \\
\hline \multicolumn{2}{|c|}{6 Empacotamento de experiências } & \\
\hline
\end{tabular}

Figura 1 - Desenho experimental do estudo de caso replicado

$\mathrm{O}$ estudo de caso é executado em três microempresas associadas à incubadora Centro GeNESS, estabelecendo em cada empresa um programa de mensuração individual com enfoque na duração, esforço e custo relacionados aos projetos de software, respeitando as características e necessidades particulares das empresas. Os programas de mensuração seguem o processo GQM [3]. Além de estabelecer os programas de mensuração, foi planejado e executado um estudo sobre os custos e benefícios relacionados à aplicação da mensuração nestes estudos de casos. O programa foi completamente planejado para as três empresas participantes dos estudos de casos. Somente uma empresa executou-o efetivamente. Nas demais empresas a continuação do programa foi inviabilizada devido problemas internos relacionados ao foco de seus projetos.

\section{Análise dos Estudos de Casos em Relação aos Custos e Benefícios}

Os custos da fase de planejamento dos programas foram medidos nas três empresas. Como duas das empresas foram impossibilitadas de prosseguir na fase de execução, os custos referentes à esta fase foram medidos em apenas uma das empresas. Os benefícios também só puderam ser vistos na empresa que deu continuidade ao programa de mensuração.

\section{Custos dos Programas de Mensuração}

Os custos do programa de mensuração são medidos através do esforço gasto (em pessoas-hora) com as atividades de mensuração. São considerados os custos da equipe GQM, responsável principalmente pela construção do plano do programa de mensuração e os custos das empresas, que têm participação na definição dos planos do programa, sua revisão e, principalmente, pela coleta de dados. $\mathrm{Na}$ figura 2 pode-se visualizar o gasto com o 
planejamento dos programas de mensuração por equipe (GQM/empresa) em cada uma das empresas participantes:

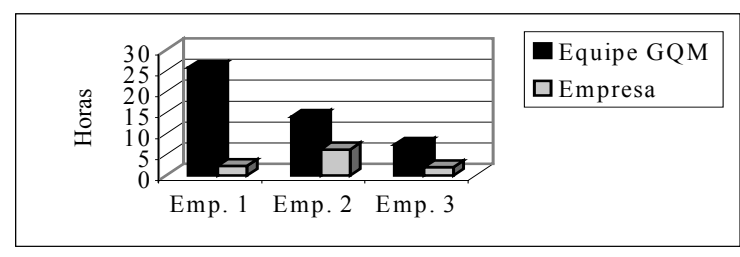

Figura 2 - Esforço do planejamento

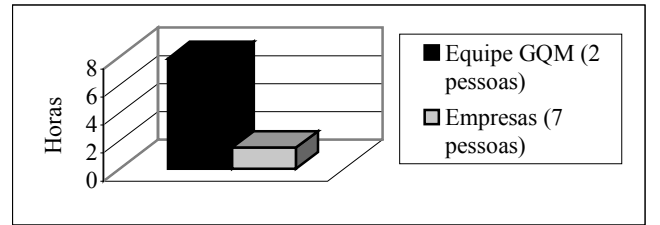

Figura 3 - Média do esforço do planejamento por pessoa

A média do esforço de planejamento (figura 3) mostra que o esforço de planejar um programa de mensuração é bem mais alto para a equipe GQM. Sem o auxílio de uma equipe de qualidade ou um modelo de mensuração, a aplicação do programa torna-se inicialmente custosa. No início do planejamento da primeira empresa não haviam programas de mensuração aplicados em microempresas com enfoque na gerência, assim, os fatores de qualidade tiveram que ser detalhados sem referências, no segundo e terceiro planos pôde-se reutilizar vários dos fatores de qualidade do primeiro resultando em uma diminuação do esforço da equipe GQM.

A equipe da empresa 2 teve um esforço mais elevado devido a continuação no programa de mensuração. As demais empresas tiveram o custo relacionado apenas às atividades da fase de planejamento, não tendo participado da última atividade desta fase (revisão do plano do programa), o que gerou um custo de planejamento mais alto para a empresa 2.

A fase de execução do programa de mensuração só se deu na empresa 2. Com início no mês de agosto, o programa continua a ser executado na empresa. Na figura 4 é apresentado o custo da execução do programa por equipe, considerando um período de quatro meses.

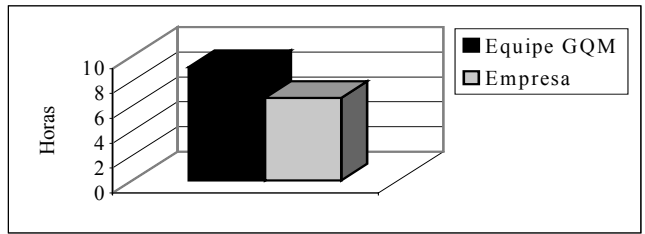

\section{Figura 4 - Esforço da execução do programa de mensuração}

Após o planejamento do programa a empresa começou a atuar mais efetivamente. Atividades como coleta e digitalização dos dados foram realizadas por membros da equipe do projeto. A participação da equipe GQM com o início da fase de execução restringiu-se à análise dos dados, organização das feedback sessions e acompanhamento do processo como um todo. Pode-se perceber que apesar do aumento no custo para a empresa, este ainda continua baixo.

\section{Benefícios do Programa}

Como o programa está sendo executado a pouco tempo não se pode fazer uma análise quantitativa sobre os benefícios alcançados, isso poderá ser melhor observado quando o projeto estiver mais avançado e a mensuração tiver sido aplicada por um período maior. Mesmo assim, vários pontos positivos da mensuração já são identificados.

Inicialmente, espera-se que, com o programa de mensuração, a empresa consiga entender aspectos relacionados a duração, o esforço e o custo de seus projetos. Identificar este entendimento é algo difícil de mensurar, principalmente numa fase tão inicial do programa. Para isso, foi desenvolvido um questionário para análise de benefícios do programa de 
mensuração baseado em GQM [2] com o qual a empresa pôde apresentar, mesmo que informalmente, alguns dos benefícios alcançados:

- Auxílio para o desenvolvimento de um modelo de processo.

- Auxílio para a definição de metas de melhoria na prática.

- Melhor monitoramento dos projetos e da empresa.

Fazendo uma análise entre os custos e os benefícios da mensuração baseado em GQM, é possível perceber que com a abordagem adaptada ao contexto, os custos tornam-se mais baixos e os benefícios advindos podem ser vistos desde o início da execução do programa.

\section{Lições Aprendidas com a Aplicação dos Programas de Mensuração}

A inviabilização do programa em duas das empresas participantes dos estudos de casos devido a mudanças no foco de seus projetos permitiu identificar uma pré-condição para a aplicação de mensuração: o contexto deve ser relativamente estável, a alteração do contexto complica a continuação da mensuração pois requer um re-planejamento. Entretanto, pequenas alterações nos planos GQM são comuns no decorrer do programa não impossibilitando o programa.

A empresa que deu continuidade ao programa, iniciou a coleta de dados baseada num modelo de processo que havia definido. Após um mês de coleta, percebeu-se que este modelo não era válido e as atividades não estavam bem definidas. No intuito de continuar a execução do programa, e também auxiliar a empresa na constituição de um modelo de processo específico, o plano GQM foi alterado adequadamente.

Algumas adaptações foram feitas no processo GQM para atender as limitações e caracteristicas específicas de microempresas: numa única reunião, com a presença de todos os sócios da empresa, foi feita a caracterização da empresa, definição e formalização da meta, ao invés de fazer reuniões separadas para cada atividade e entrevistas individuais; desenvolvimento de modelos no plano GQM, definindo já a análise de dados em detalhes, facilitando e diminuindo o esforço desta fase.

A partir das lições aprendidas na execução dos estudos de caso, e também da diminuição do custo de se aplicar mensuração baseada em GQM a partir deste processo adaptado, percebe-se a necessidade de se desenvolver um modelo adaptado ao contexto de microempresas. Este modelo deve enfocar nos problemas gerenciais destas empresas - falta de um modelo de processo definido, inexperiência na área de gerenciamento de projetos, entre outros - e também respeitar a falta de recursos desse setor. A partir de um modelo com estas características a aplicação de programas de mensuração baseados em GQM é viabilizada no contexto de microempresas, permitindo que estas empresas estabeleçam um controle sobre os processos gerenciais.

\section{Modelo de Mensuração para Suporte da Gerência de Projetos em Microempresas de Software}

A partir dos programas de mensuração é desenvolvido um modelo de mensuração para gerência de projetos em microempresas de software baseado na abordagem GQM. O modelo é construído comparando e generalizando as experiencias concretas das empresas participantes no estudo de caso. 
O objetivo deste modelo é dar suporte a microempresas para a aplicação de um programa de mensuração baseado na abordagem GQM enfocando na meta: "Entender a duração, o esforço e o custo do projeto de software sob o ponto de vista do gerente de projeto na empresa X". Para aplicar um programa de mensuração com o suporte deste modelo, a empresa seguirá todos os passos de um programa de mensuração baseado em GQM [3]. Para cada atividade a ser realizada o modelo fornece elementos relevantes e diretrizes para adaptação das mesmas. Quando aplicado, o modelo pode ser utilizado como uma base para o programa de mensuração em uma empresa específica, necessitando ser revisado para adaptção ao contexto específico, ao invés de iniciar o desenvolvimento do programa do zero.

Seguindo o processo GQM [3], o ponto inicial deste modelo é o abstraction sheet, representando a união dos abstraction sheets das empresas participantes no estudo e outros fatores de qualidade com base na literatura [5]. A figura 5 apresenta uma parte do abstraction sheet:

\begin{tabular}{|l|l|c|c|c|}
\hline \multicolumn{1}{|c|}{ Objeto } & Objetivo & Enfoque de Qualidade & Ponto de Vista & Contexto \\
\hline Projeto & Entender & Duração, esforço e custo & Gerente de projeto & Empresa X \\
\hline \multicolumn{5}{|c|}{ Fatores de Qualidade } \\
\hline $\begin{array}{l}\text { Duração: Duração total por projeto } \\
\text { Esforço: Esforço total do projeto por mês }\end{array}$ & Custo: Custo total por projeto \\
\hline \multicolumn{4}{|c|}{ Custo por fase do projeto } \\
\hline $\begin{array}{l}\text { De acordo com os fatores de qualidade selecionados deverão ser feitas hipóteses para a formação de uma linha base. } \\
\text { Exemplo de hipóteses são: - O projeto durará 10 meses; - A atividade 2.1 exigirá um esforço de 50 hs. }\end{array}$ \\
\hline
\end{tabular}

\section{Figura 5 - Abstraction sheet do modelo}

Para cada fator de qualidade do abstraction sheet do modelo são derivadas perguntas e modelos genéricos. São definidas as medidas a serem coletadas para cada pergunta e o plano de mensuração contendo as informações necessárias sobre todas as medidas. A figura 6 apresenta a estrutura de uma pergunta/modelo:

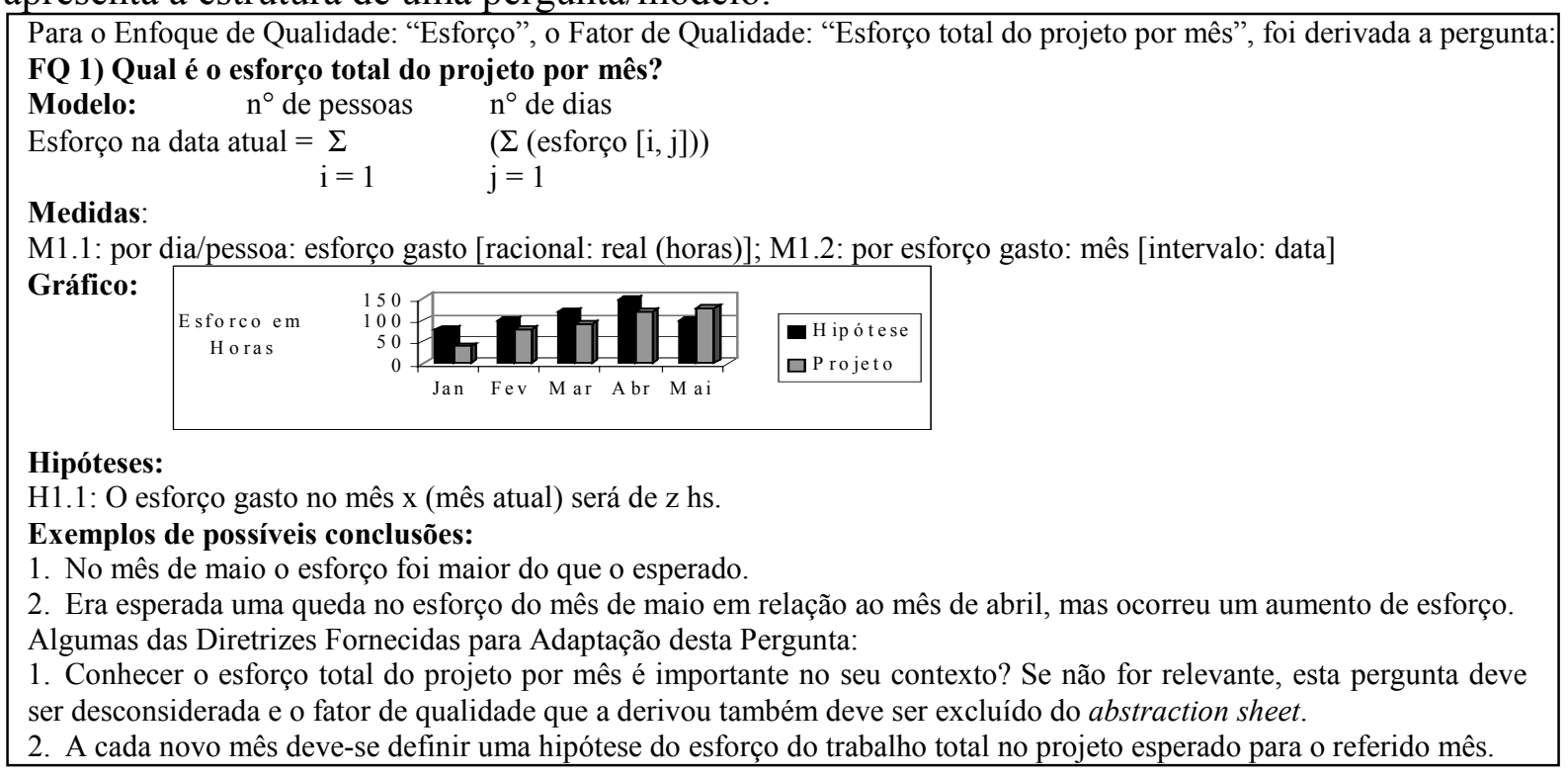

\section{Figura 6 - Exemplo de Pergunta/Modelo e Sua Estrutura}

Com base no plano GQM é desenvolvido o plano de coleta de dados. O modelo oferece todos os procedimentos de coleta de dados para as medidas definidas no modelo de mensuração e dos instrumentos criados para a coleta de dados.

$\mathrm{Na}$ figura 7 é apresentado um procedimento, correspondente a uma das medidas derivadas dos modelos gerados na pergunta exemplo utilizada anteriormente: 


\begin{tabular}{|c|c|c|c|c|c|}
\hline $\mathbf{N}^{\circ}$ Proc. & Medidas Associadas & Descrição & Tempo & Papel & Instrumento \\
\hline 1 & M1.1 & $\begin{array}{l}\text { Esforço gasto por dia / } \\
\text { por pessoa }\end{array}$ & \begin{tabular}{|l}
$\begin{array}{l}\text { Periodicamente: } \\
\text { diariamente }\end{array}$ \\
\end{tabular} & Desenvolvedores & $\begin{array}{l}\text { Formulários para } \\
\text { Coleta de Dados }\end{array}$ \\
\hline $\begin{array}{l}\text { Algumas d } \\
\text { 1.Pela iden } \\
\text { 2.Para cada } \\
\text { 2.1. Medi }\end{array}$ & $\begin{array}{l}\text { diretrizes para adaptaç } \\
\text { icação das medidas (M } \\
\text { hedida verifique se seu } \\
\text { s Associadas: As medi }\end{array}$ & $\begin{array}{l}\text { o fornecidas para os proc } \\
\text { x.y) selecione-as na tabel } \\
\text { dados são pertinentes. E } \\
\text { las que não estão associa }\end{array}$ & $\begin{array}{l}\text { edimentos são: } \\
\text { do plano da cole } \\
\text { n caso de alteraç } \\
\text { las a nenhuma pe }\end{array}$ & $\begin{array}{l}\text { de dados. } \\
\text { proceda como s } \\
\text { inta devem ser de }\end{array}$ & $\begin{array}{l}\text { e: } \\
\text { onsideradas. }\end{array}$ \\
\hline
\end{tabular}

\section{Figura 7 - Exemplo de Procedimento do Plano de Coleta de Dados}

Para cada medida é explicitado qual o instrumento a ser utilizado para sua coleta. $\mathrm{O}$ modelo de mensuração também presta suporte para a criação destes instrumentos. Além disso, é explicado como eles devem ser utilizados e é apresentado um exemplo genérico. Por exemplo, o formulário para coleta de dados (figura 8), é preenchido diariamente por todos os membros da equipe envolvidos no projeto que se está analisando.

\begin{tabular}{|c|c|c|c|c|}
\hline \multicolumn{5}{|c|}{$\begin{array}{l}\text { Formulário para Coleta de Dados } \\
\text { Empresa: Nome da empresa em que o programa será aplicado } \\
\text { Nome: Nome do coletor que utilizará este formulário }\end{array}$} \\
\hline $\mathrm{D}$ & \multicolumn{2}{|c|}{ Fase 1: Planejamento } & \multicolumn{2}{|c|}{ Fase 2: Desenvolvimento } \\
\hline ia & Ativ. 1.1 & Ativ. 1.2 & Ativ. 2.1 & Ativ. 2.2 \\
\hline \multicolumn{5}{|l|}{1} \\
\hline$\ldots$ & & & & \\
\hline \multicolumn{5}{|c|}{$\begin{array}{l}\text { Algumas das diretrizes para adaptação fornecidas para a criação deste formulário são: } \\
\text { 1. O formulário deve ser adaptado às atividades da empresa. Deve-se acrescentar colunas de acordo com o número } \\
\text { de fases/atividades... em que o projeto é dividido. } \\
\text { 2. É importante que os campos estejam bem organizados para facilitar a coleta de dados. }\end{array}$} \\
\hline
\end{tabular}

\section{Figura 8 - Formulário para Coleta de Dados}

Os estudos de caso realizados foram feitos em projetos ainda em fase inicial por isso não chegaram à etapa de empacotamento das experiências adquiridas. $\mathrm{O}$ modelo de mensuração apresenta uma captura de experiências baseada na literatura [7]. A captura das experiências obtidas com o programa de mensuração é feita no final do projeto, quando se tem todos os dados necessários para entende-lo como um todo. Estas informações são guardadas num documento específico que contém a identificação do projeto, os dados relevantes capturados e as lições aprendidas com a análise destes dados. Este documento pode ser utilizado mais tarde quando um novo projeto, similar ao anterior, for desenvolvido. Com os dados obtidos pode-se prever melhor como será o andamento deste novo projeto, pois estará se baseando num projeto real, desenvolvido dentro do mesmo contexto.

\section{Vantagens de Utilizar o Modelo de Mensuração Baseado em GQM}

O método GQM tradicional [9,3] não oferece nenhum suporte específico para o planejamento e execução de um programa de mensuração em microempresas de software. Isso devido ao fato da abordagem GQM ser um método genérico para o planejamento e execução de programas de mensuração sem o uso de modelos e métricas pré-definidas.

Dentro do contexto de microempresas, este é um fator complicante para sua aplicação, pois desta forma o GQM exige bastante esforço e experiência para ser aplicado efetivamente, o que falta nestas empresas. Sendo assim, o modelo desenvolvido neste trabalho enfoca especificamente nas características, problemas e limitações das microempresas de software e é direcionado a uma meta específica: a avaliação de aspectos gerenciais, tais como, a duração, o esforço e o custo. Desta forma, o modelo oferece uma melhor pré-condição para o estabelecimento de mensuração também neste tipo de empresa. Ainda assim, o modelo 
precisa ser adaptado a um contexto específico. Para isso são oferecidas diretrizes explícitas indicando as adaptações mais prováveis de serem necessárias seguindo o processo GQM.

Como já observou-se uma diminuição no esforço de planejamento pela reutilização informal dos planos desenvolvidos no estudo de caso, esperamos que o modelo permita reduzir o esforço necessário para a aplicação do programa de mensuração e para melhorar a qualidade do programa desenvolvido.

\section{Conclusão}

Neste trabalho mostra-se que é possível customizar e aplicar mensuração de software também em microempresas de software. Isto é apresentado a partir de um estudo de caso repetido aplicado em três microempresas incubadas no Centro GeNESS. A execução de mensuração no contexto estudado se mostrou de grande eficiência, pois gerou vários benefícios que são do interesse da empresa com um custo baixo para a mesma.

A partir destes três estudos de caso foi desenvolvido o modelo de mensuração para gerência de projetos em microempresas de software com enfoque na duração, esforço e custo dos projetos. Além da diminuição dos custos, o modelo pretende aumentar a qualidade do programa de mensuração. Desta forma, espera-se que o modelo desenvolvido forneça às microempresas de software um suporte para aplicação de mensuração voltada à gerência de projetos a partir de um mecanismo efetivo para o planejamento e controle de projetos de software. Essas melhorias ainda precisam ser validadas através de novos estudos casos, aplicando o modelo desenvolvido em microempresas de software.

\section{Bibliografia}

[1] A. Anacleto e C. Gresse von Wangenheim. Modelo de Mensuração para Gerência de Projetos em Microempresas de Software. Relatório Técnico GeNESS006.01P, Centro GeNESS, 2001.

[2] A. Anacleto e C. Gresse von Wangenheim. Questionário para Análise dos Beneficios do Programa de Mensuração Baseado em GQM. Relatório Técnico GeNESS005.01P, Centro GeNESS, 2001.

[3] C. Gresse von Wangenheim, H. D. Rombach e G. Ruhe. Tutorial on Melhoramento de Software Baseado em Mensuração - Como Aplicar GQM na Prática?. Proceedings of the IX CITS, Curitiba, Brazil, 1998.

[4] H. D. Rombach et al. Experimental Software Engineering Issues: A Critical Assessment and Future Directions. Lecture Notes in Computer Science Nr. 706, 1993.

[5] I. Sommerville. Software Engineering. Fifth Edition, Addison-Wesley, 1996

[6] Ministério de Ciência e Tecnologia (MCT). Pesquisa Nacional de Qualidade e Produtividade no Setor de Software Brasileiro. Comunicação pessoal. Brasília, 1999.

[7] Software Measurement Guidebook. Technical Report SEL-94-002, NASA Goddard Space Flight Center, Greenbelt MD 20771, July 1994.

[8] V.R. Basili, G. Caldiera, H.D. Rombach. Experience Factory. In John J. Marciniak, ed., Encyclopedia of Software Engineering, vol.1. John Wiley \& Sons, 1994.

[9] V.R. Basili, G. Caldiera, H.D. Rombach. Goal/Question/Metric Approach. In John J. Marciniak (ed.), Encyclopedia of Software Engineering, vol.1. John Wiley\&Sons, 1994. 